\title{
Paraplegia
}

\section{Serum Alkaline Phosphatase and Inorganic Phosphorus Values in Spinal Cord Injury Patients with Heterotopic Ossification}

\author{
S. W. Kim, MD, ${ }^{1}$ R. A. Charter, PhD,${ }^{3}$ C. J. Chai, MD, ${ }^{2}$ S. K. Kim, DPh, ${ }^{4}$ \\ E. S. Kim, $\mathbf{M D}^{2}$ \\ ${ }^{1}$ Spinal Cord Injury Service, Department of Veterans Affairs Medical Center, Bronx, \\ New York, ${ }^{2}$ Spinal Cord Injury Service, ${ }^{3}$ Psychology Service, ${ }^{4}$ Pharmacy Service, \\ Long Beach, California, USA.
}

\section{Summary}

The blood chemistry was studied in 140 spinal cord injury (SCI) patients (acute injury ward), including 18 patients who developed heterotopic ossification (HO). Comparisons between the $\mathrm{HO}$ and non-HO groups were made to determine if the alkaline phosphatase $(A P)$, inorganic phosphorus $(P)$, or calcium $(C a)$ levels were of diagnostic value. The results showed that $A P, P$, and $C a$ by themselves were of little help in the diagnosis of HO. However, the combination of elevated $A P$ and $P$ was significant, especially if both were consistently elevated. There were no significant differences between the $\mathrm{HO}$ and non-HO groups concerning completeness or level of spinal injury.

Key words: Spinal cord injury; Heterotopic ossification; Alkaline phosphatase; Inorganic phosphorus

Although some research workers have pointed out that an increased alkaline phosphatase (AP) value is not necessarily associated with heterotopic ossification (HO) (Rossier et al., 1973; Bergmann et al., 1977; Orzel and Rudd, 1985; Stover, 1986), it has been suggested that an elevation of AP is the most reliable indicator of $\mathrm{HO}$ in spinal cord injury (SCI) patients.

Several studies have also examined serum calcium $(\mathrm{Ca})$ and phosphorus $(\mathbf{P})$ levels in SCI patients and found them to be increased during the first 3 months following SCI (Chantraine et al., 1970, 1971; Kaplan et al., 1978; Bergmann et al., 1977). Rarely, a relationship between serum $\mathrm{P}$ levels and $\mathrm{HO}$ has been demonstrated (Rossier et al., 1973).

This study investigates SCI patients with and without $\mathrm{HO}$ in terms of differences in serum levels of $\mathrm{Ca}, \mathrm{P}$, and AP. It also examines the temporal relationship between these levels and various forms of bony injury, including bone fracture and bone surgery. These data could permit timelier and more accurate diagnosis of $\mathrm{HO}$, and in addition, could provide further insights into the evolution of this disease. 


\section{Methods}

The subjects were 140 hospitalised SCI patients in an acute injury ward. Eighteen were diagnosed by radiography or bone scan as having $\mathrm{HO}$, whereas 122 showed no evidence of the presence of such a condition. There were 137 males and 3 females. The age range was 18 to 83 years with a mean of $37 \cdot 9$. There were 87 patients with a cervical injury (including 9 with $\mathrm{HO}: 10 \cdot 3 \%$ ), 39 with a thoracic injury (including 9 with HO: $23 \cdot 1 \%$ ), and 14 with a lumbar injury (none of which showed evicence of HO: $0 \cdot 0 \%$ ). Sixty six had complete injuries (including 10 with HO: $15 \cdot 2 \%$ ) and 74 had incomplete injuries (including 8 with HO: $10 \cdot 8 \%$ ). Those who were treated with disodium etidronate (EHDP) before obtaining blood samples were excluded from the study.

Serum AP was measured by the automated method of Bessy, Lawry and Brock (1983). Normal levels were considered to be between 30 and $115 \mathrm{mu}$ per $\mathrm{ml}$. Inorganic $\mathrm{P}$ was measured in a Technicon Auto Analyzer system. Normal levels are 2.5 to $4.5 \mathrm{mg}$ per dl. Ca was measured by Gitelman's modification of the automated procedure of Kessles and Wolfman (1983). Normal levels are 8.5 to $10.5 \mathrm{mg}$ per $\mathrm{dl}$.

Chi-square analysis was used to analyse the data, which were best represented by percentages, and analysis of variance was used to determine differences between means. The evolution of $\mathrm{HO}$ was divided into four phases: phase 1, date of injury to 4 weeks post-injury; phase 2, 4 to 8 weeks post-injury; phase 3,8 weeks to 3 months post-injury; and phase 4 , more than 3 months post-injury.

\section{Results}

There was a significant difference between the percentage of patients with $\mathrm{HO}$ at the three levels of SCI injury $\left(\chi^{2}[2]=6 \cdot 19, \mathrm{p}=0 \cdot 045\right)$. Post hoc comparisons showed non-significant differences between the cervical $(10.3 \%)$ and thoracic $(23 \cdot 1 \%)$ groups; a significant difference between the cervical and lumbar $(0 \cdot 0 \%)$ groups; and a significant difference between the thoracic and lumbar groups. There was a non-significant difference $\left(\chi^{2}[1]=0.59, \mathrm{p}=0.443\right)$ between the percentage of patients with $\mathrm{HO}$ having complete injuries $(15 \cdot 2 \%)$ and those with HO having incomplete injuries $(10 \cdot 8 \%)$.

Table I shows the percentages of $\mathrm{HO}$ and non-HO patients having at least one blood test showing abnormal levels of AP, $\mathrm{Ca}$, or P.

Table II shows the percentage of $\mathrm{HO}$ patients showing various combinations of abnormal AP, $\mathrm{Ca}$, and $\mathrm{P}$ levels. Chi-square analysis revealed non-significant differences between the $\mathrm{HO}$ and non-HO groups.

Table III shows the percentages of $\mathrm{HO}$ and non-HO patients with abnormal AP and/or P levels for the fracture/bone surgery and non-fracture/bone surgery groups. Chi-square analysis revealed that fracture/bone surgery raises both the AP and/or $P$ levels above those without fracture/bone surgery. However, the effects of fracture/bone surgery on $\mathrm{AP}$ and $\mathrm{P}$ are the same for those with $\mathrm{HO}$ and without HO.

The relationship between phase of injury and level and completeness is shown in Table IV. There was no statistically significant relationship between any of these factors. The development of $\mathrm{HO}$ in our sample was distributed across phases 
Table I Percentage of $\mathrm{HO}$ and non-HO patients having at least one blood test resul ting in levels of $\mathrm{AP}, \mathrm{P}$, or $\mathrm{Ca}$ bey ond normal limits

\begin{tabular}{crr}
\hline Test & Non-HO & HO \\
\hline AP $>115 \cdot 0$ & 63.9 & $44 \cdot 4$ \\
$\mathrm{AP}<30 \cdot 0$ & $4 \cdot 1$ & $0 \cdot 0$ \\
$\mathrm{Ca}>10 \cdot 5$ & $16 \cdot 4$ & $5 \cdot 6$ \\
$\mathrm{Ca}<8.5$ & $35 \cdot 2$ & $22 \cdot 2$ \\
$\mathrm{P}>4.5$ & $86 \cdot 9$ & $83 \cdot 3$ \\
$\mathrm{P}<2.5$ & $10 \cdot 7$ & 0.0 \\
\hline
\end{tabular}

Table II Percentage of $\mathrm{HO}$ and non-HO patients with combinations of AP and $\mathrm{P}, \mathrm{AP}$ and $\mathrm{Ca}$, or $\mathrm{AP}, \mathrm{P}$, and $\mathrm{Ca}$ elevated

\begin{tabular}{lcr}
\hline Electrolytes & Non-HO & HO \\
$\mathrm{AP}>115$ and $\mathrm{P}>4 \cdot 5$ & $-53 \cdot 4$ & $61 \cdot 1$ \\
$\mathrm{AP}>115$ and $\mathrm{P}<2 \cdot 5$ & $4 \cdot 1$ & $0 \cdot 0$ \\
$\mathrm{AP}>115$ and $\mathrm{Ca}>10 \cdot 5$ & $4 \cdot 1$ & $5 \cdot 6$ \\
$\mathrm{AP}>115$ and $\mathrm{Ca}<8.5$ & $14 \cdot 8$ & $5 \cdot 6$ \\
$\mathrm{AP}>115$ and $\mathrm{Ca}>4.5$ and $\mathrm{P}>4.5$ & $2 \cdot 5$ & $0 \cdot 0$ \\
\hline
\end{tabular}

Table III Relationship between fracture/bone surgery, HO and non-HO, elevated AP and/or P

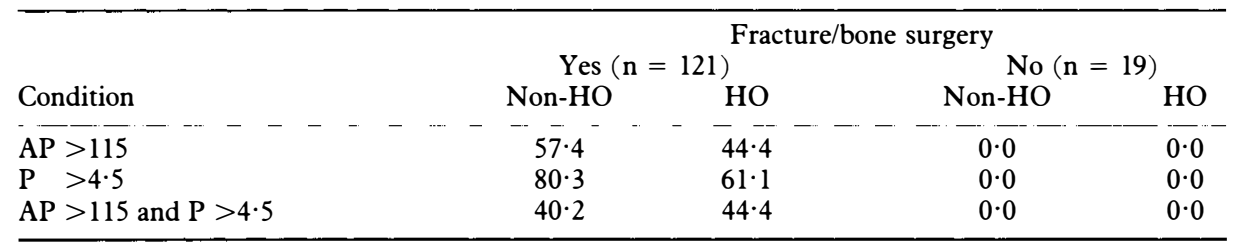

Table IV Relationship between phase, level of injury, and complete or incomplete injury for those patients with $\mathrm{HO}$

\begin{tabular}{|c|c|c|c|c|c|c|}
\hline \multirow[b]{2}{*}{ Phase } & \multicolumn{2}{|c|}{ Cervical } & \multicolumn{2}{|c|}{ Thoracic } & \multicolumn{2}{|c|}{ Lumbar } \\
\hline & Complete & Incomplete & Compl ete & Incomplete & Complete & Incompl ete \\
\hline 1 & $5 \cdot 6$ & $5 \cdot 6$ & $5 \cdot 6$ & $5 \cdot 6$ & $0 \cdot 0$ & $0 \cdot 0$ \\
\hline 2 & 0.0 & $16 \cdot 7$ & $16 \cdot 7$ & $5 \cdot 6$ & 0.0 & 0.0 \\
\hline 3 & $5 \cdot 6$ & $11 \cdot 1$ & $16 \cdot 7$ & $0 \cdot 0$ & $0 \cdot 0$ & $0 \cdot 0$ \\
\hline 4 & $5 \cdot 6$ & $0 \cdot 0$ & 0.0 & $0 \cdot 0$ & 0.0 & 0.0 \\
\hline
\end{tabular}

as follows: phase 1,4 patients $(22 \%)$; phase 2,7 patients $(39 \%)$; phase 3,6 patients $(33 \%)$; and phase 4,1 patient $(6 \%)$. The difference in percentages was not statistically significant. It should be noted that $\mathrm{HO}$ was not found in any of our patients with lumbar lesions.

Figure 1 shows the relationship between the likelihood of the development of $\mathrm{HO}$ and the proportion of blood tests showing elevated AP levels $(>115)$. This likelihood, as measured by the difference in percentages between the $\mathrm{HO}$ and non- 
HO patients, increased greatly if half or more of the blood levels of AP were elevated.

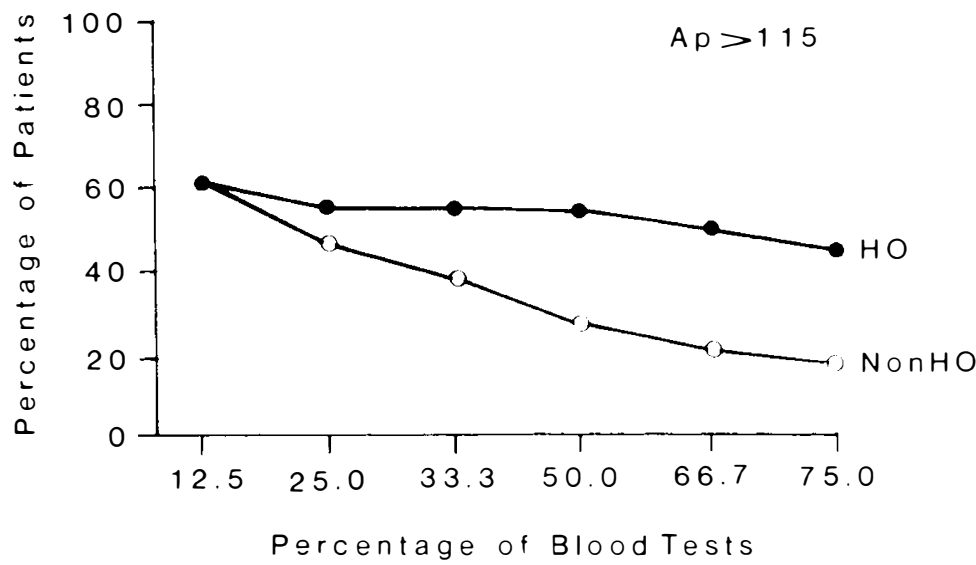

Figure 1 Percentage of $\mathrm{HO}$ and non-HO patients having AP $>115$ at 6 different percentages of blood tests.

Figure 2 shows the relationship between the likelihood of the development of $\mathrm{HO}$ and the proportion of blood tests showing elevated P levels $(>4 \cdot 5)$. There were no significant differences between the percentages of $\mathrm{HO}$ and non-HO patients at any of the percentages of blood tests. The likelihood of developing HO appeared to bear no relation to the proportion of blood tests showing elevated $\mathrm{P}$ levels.

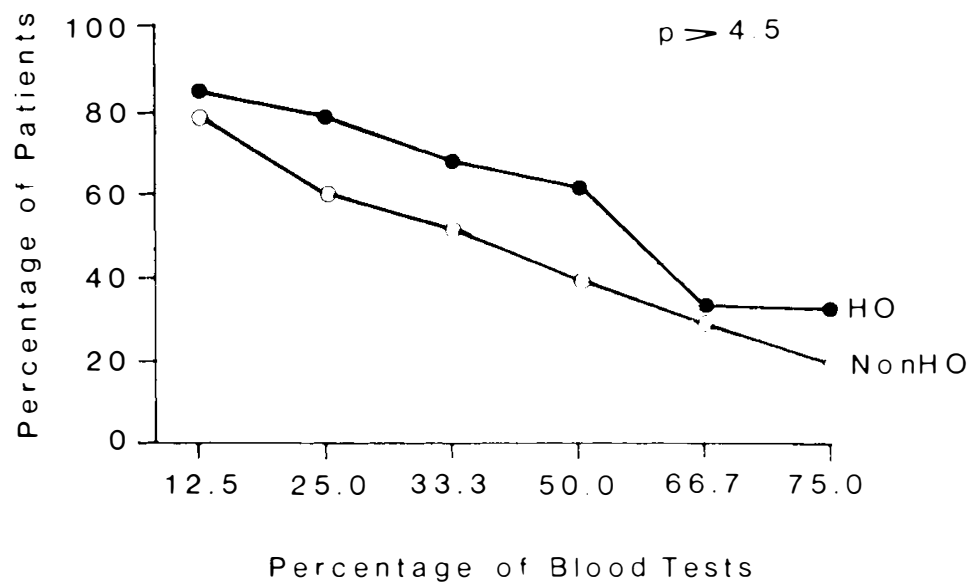

Figure 2 Percentage of $\mathrm{HO}$ and non-HO patients having $\mathrm{P}>4.5$ at 6 different percentages of blood tests.

Figure 3 shows the relationship between the likelihood of developing $\mathrm{HO}$ and the proportion of blood tests showing elevation of both AP $(>115)$ and $\mathrm{P}(>4 \cdot 5)$. The difference between the percentage of $\mathrm{HO}$ and non- $\mathrm{HO}$ patients was significant at all percentages of blood tests. The likelihood of developing $\mathrm{HO}$ rose significantly as the proportion of tests showing elevation of both values increased. 


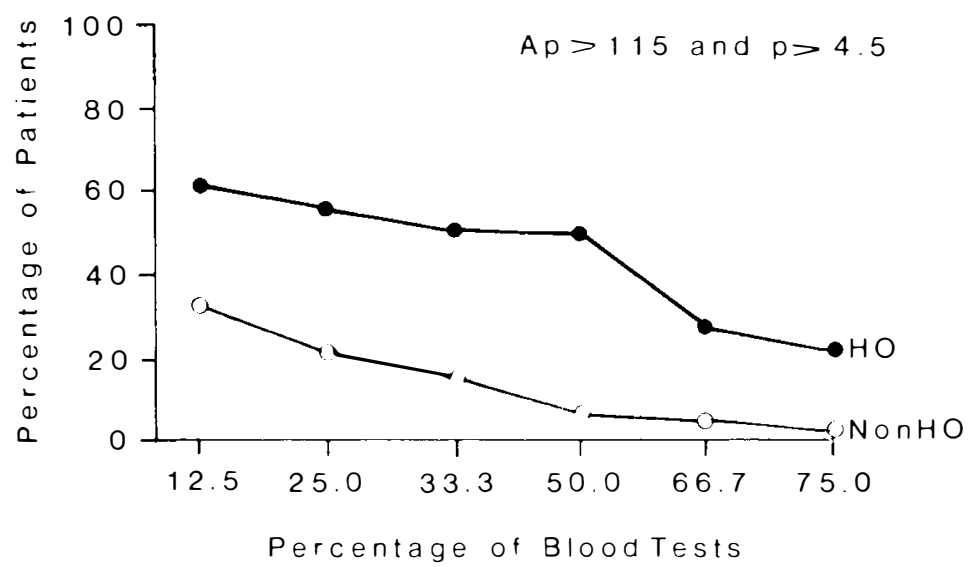

Figure 3 Percentage of $\mathrm{HO}$ and non-HO patients having AP $>115$ and $\mathrm{P}>4.5$ at 6 different percentages of blood tests.

\section{Discussion}

The aetiology of HO remains unknown. Researchers have hypothesised various aetiologies, including biochemical factors (Farley and Baylink, 1982; Maugh, 1982), mesenchymal metaplasia (Ostrowski and Wlodarski, 1971), bone metabolism (Chantraine, 1971; Bergmann et al., 1978), and trauma to joints (Silver, 1969; Kaplan et al., 1978, Izumi, 1983).

It is well known that the major portion of serum AP originates from osteoblasts and chondroblasts. However, conflicting results have been obtained relative to serum AP levels in patients with HO (Kline, 1966; Nicholos, 1973; Orzel et al., 1985; Bergmann et al., 1977). This may be due in part to the presence of additional variables, especially the presence or absence of a fracture or bone surgery. At times consistent elevation of AP may be associated with $\mathrm{HO}$ that is spreading extensively over the entire joint, causing severe ankylosis (Orzel et al., 1985; Rossier et al., 1973). The finding of increased serum $P$ during the period of bone resorption is compatible with an increase in the number of osteoblasts.

The data we have collected suggest that the relationship between AP, P, or Ca values alone and $\mathrm{HO}$ is not statistically significant (Table I), and that elevation of $\mathrm{AP}$ and $\mathrm{P}$ together, based on one blood test, occurred more often in patients with $\mathrm{HO}$ than in those without HO (Table II), but the difference was not statistically significant. Until now the diagnostic significance of elevated levels of both AP and $\mathrm{P}$ in $\mathrm{HO}$ has been studied.

It might be presumed that fracture or bone surgery (e.g. spinal fusion) may be associated with elevated blood levels of $\mathrm{AP}$ and $\mathrm{P}$, but our findings failed to provide evidence of a relationship between fracture and HO. This seems to suggest that $\mathrm{AP}$ and $\mathrm{P}$ are more related to the development of $\mathrm{HO}$ than fracture or bone surgery.

The literature describes different time periods ranging from 19 days to 4 months for the development of HO following SCI (Silver, 1969; Venier et al., 1971; Stover, 1986). The development of $\mathrm{HO}$ in our sample was distributed across phases as follows: phase 1, 4 patients (22\%); phase 2, 7 patients (39\%); phase 3, 6 patients 
(33\%); and phase 4,1 patient $(6 \%)$. The difference in percentages was not statistically significant.

Our findings show no significant relationship between $\mathrm{HO}$ and the completeness of injury. We found a non-significant difference between the percentage of patients with $\mathrm{HO}$ in the cervical $(10 \cdot 3 \%)$ and thoracic $(23 \cdot 1 \%)$ groups. However, significant differences were found between the cervical and lumbar $(0.0 \%)$ and the thoracic and lumbar groups. Our results, therefore, differ from those of Damanski (1961), who reported that the peak incidence of $\mathrm{HO}$ was at thoracic spinal levels, and from those of Knudsen et al. (1982), who found that those with HO were more likely to have complete lesions.

The present study does not totally clarify the role of AP as a diagnostic tool for determining the presence of $\mathrm{HO}$. AP did increase in $44.4 \%$ of our patients with $\mathrm{HO}$, but it was also elevated in $63.9 \%$ of those without HO. Figure 1, however, shows that a higher percentage of blood tests revealing elevated AP levels is associated with a greater likelihood that $\mathrm{HO}$ has developed. Approximately 50\% of patients with $\mathrm{HO}$ showed elevated AP levels $50 \%$ or more of the time, whereas this was true in only about $20 \%$ of non-HO patients.

Our results confirm the results of Rossier et al. (1973), that is, that serum P determinations, when evaluated alone, are of little value in the diagnosis of HO. Table I and Figure 3 show, and the statistical analysis confirms, that there are no differences between the $\mathrm{HO}$ and non-HO groups in the percentage of patients with elevated $P$.

Our study reveals, however, that serum $\mathrm{P}$ can be of diagnostic value when evaluated with AP. Table II shows that $61 \cdot 1 \%$ of our HO sample had elevations of both AP and $\mathrm{P}$ and $43.4 \%$ of the non-HO group had elevations of both values. This difference was statistically non-significant and, in practical terms, is not very large, and may therefore be of limited diagnostic value. Figure 3, however, further amplifies the value of increased AP and $\mathrm{P}$ in the diagnosis of HO. This Figure shows that as the proportion of blood tests showing both elevated AP and P levels rises, the likelihood that the patient has $\mathrm{HO}$ increases. Only about $5 \%$ of the nonHO patients showed elevations of both AP and P on $50 \%$ or more of their blood tests, while this was true in approximately $30 \%$ of those with $\mathrm{HO}$. Although having normal serum levels of both AP and $\mathrm{P}$ does not rule out the presence of $\mathrm{HO}$, persistent elevation of both values greatly increases the likelihood that the patient has HO.

\section{References}

Chantraine A 1970-71 Clinical investigation of bone metabolism in spinal cord lesions. Paraplegia 8:253-259

Chantraine A, Heyney NG, Franchimont P 1979 Bone metabolism, parathyroid hormone, and calcitonin in paraplegia. Calcification Tissue International 27:199-204

Bergmann P, Heilman A, Schoutens A, Paternot J, Tricot A 1977-78 Longitudinal study of calcium and bone metabolism in paraplegic patients. Paraplegia 15:147-150.

DAMANSKI M 1961 Heterotopic ossification in paraplegia: a clinical study. Fournal of Bone and foint Surgery 43B:286-299

FARLEY JR, BAYLINK DJ 1982 Purification of a skeletal growth factor from human bone. Biochemistry 21:3502-3507

Heath HI iII, Schaaf M, Wray HL, Monchik JM, Garll JM 1973 In: Frame B (ed) Clinical Aspects of Metabolic Bone Disease. Excerpta Medica, Amsterdam.

IzUMI K 1983 Study of ectopic bone formation in experimental spinal cord injured rabbits. Paraplegia 6:351-363 
Kaplan PE, Gandhavadi B, Richards L, Goldschmidt J 1978 Calcium balance in paraplegic patients: influence of injury duration and ambulation. Archives of Physical Medicine and Rehabilitation Medicine 59:447-450

KLINe L, van DEN Noort S, PETAK J 1966 Sequential studies of urinary hydroxyproline and serum alkalinephosphatase in acute paraplegics. Med Serv Canada 22:24-533

KNUDSEN L, LUNDBERG D, ERICSSON G 1982 Myositis ossificans circumscripta in para/tetraplegics. Scandanavian fournal of Rheumatology 11:27-31

MAUGH TH 1982 Human skeletal growth factor isolated. Science 217:819

Nicholos J 1973 Ectopic bone formation in patients with spinal cord injury. Archives of Physical Medicine and Rehabilitation 54:354-359

ORZEL J, RUDD TG 1985 Heterotopic bone formation: clinical laboratory and imagery correlation. Fournal of Nuclear Medicine 26: 125-132

OSTROWSKI K, WLODARSKI K 1971 Induction of heterotopic bone formation. In: Comar CL, Bronner J (eds) Biochemistry and Physiology of Bone, Vol III. Academic Press, New York pp 3502-2507

Rossier AB, BusSAT PH et al. 1973 Current factors on para-osteo-arthropathy (POA). Paraplegia 11:36-78

SILVER JR 1969 Heterotopic Ossification: A clinical study of its possible relationship to trauma. Paraplegia 7:220-230

STOVER SL 1986 Management of Spinal Cord Injuries. Williams \& Wilkins, Baltimore, pp 524-533

VENIER LH, DituNNo JR JF 1971 Heterotopic ossification in the paraplegic patient. Archives of Physical and Medical Rehabilitation 52:475-479 